\title{
Identification and Sequencing of Genotype VII of Newcastle Disease Virus From Chicken Flocks In Six Egyptian Governorates
}

\author{
Hagar M. Ahmed*, M.M. Amer**, Kh. M. Elbayoumi*, S.A. Amer*, M. A. \\ Kutkat* \\ * Department of Poultry Diseases, Veterinary Research Division, National Research \\ Centre "Department of Poultry Diseases, Faculty of Veterinary Medicine, Cairo \\ University, Cairo, Egypt
}

\begin{abstract}
NEWCASTLE disease (ND) is a very contagious disease in chickens and turkeys 1 and one of the most important diseases of poultry in the world. The infection causes sudden death with high mortality. ND in recent years showed symptoms more severe than previous symptoms of this disease. NDV infection of genotype VII has been reported to cause this outbreak in several commercial poultry farms. In this study the isolated viruses were molecularly characterized by RT-PCR targeting the partial F-gene of NDV. Partial F gene sequence analysis showed that the isolated NDV strains belong to genotype VII with the characteristic amino acid sequences of the F0 protein proteolytic cleavage site motifs $\left({ }^{112} \mathrm{RRQKRF}{ }^{117}\right)$ for the velogenic NDV (vNDV) strains. From the isolated and molecular identified NDV Genotype VII four selected isolates (NDV/Chicken/EG-MN/NRC/2015, NDV/Chicken/EGQU/NRC/2015, NDV/Chicken/EG-SH/NRC/2015 and NDV/Chicken/EG -SH2/ NR/2015) were sequenced for NDV F gene and nucleotide sequences were submitted to GenBank and given accession numbers (MF418017), (MF418018), (MF418019) and (MF418020); respectively.
\end{abstract}

Though no significant genetic changes observed, the multiple vNDV outbreaks in vaccinated chicken require continuous monitoring of the evolution and the epidemiology characteristics of the VNDV as well as evaluation of the used vaccines.

Keywords: Identification, Genotype VII, Sequencing, Newcastle disease virus, Chicken.

\section{Introduction}

Newcastle disease (ND) is a highly contagious viral disease causing high mortality and severe disease in birds especially chickens as the most susceptible host [1]. Newcastle Disease Virus (NDV), is classified in the genus Avulavirus, subfamily Paramyxovirinae, family Paramyxoviridae, order Mononegavirales [2]. The genome contains six major genes encoding the nucleocapsid protein (NP), phosphoprotein (P), matrix (M) protein, fusion (F) protein, haemagglutininneuraminidase $(\mathrm{HN})$ and RNA-dependent RNA polymerase (L) protein in the order of $3 /-\mathrm{N}-\mathrm{P}-\mathrm{M}-$ F-HN-L-5' [3].

Infection of the disease varies from subclinical to severe or systemic infection with high mortality rate depending on the virulence of virus strain and the host susceptibility. Based on the clinical signs in chicken, the NDV are categorized into five pathotypes including viscerotropic velogenic, neurotropic velogenic, mesogenic, lentogenic respiratory and asymptomatic enteric [4]. Genetic diversity between NDV strains was proved using DNA sequencing and phylogenetic analysis of either complete or partial nucleotide sequences of the $\mathrm{F}$ gene. The amino acid sequence of the F0 precursors in NDV of low virulence (loNDV) are characterized by a monobasic amino acid sequence motif at the $\mathrm{C}$-terminus of the F2 protein and a leucine at the $\mathrm{N}$-terminus of the F1 protein, 112G-R/K-Q-G-R-L117. The F0 precursors of loNDV are cleaved only extracellularly by trypsin-like proteases present in the respiratory and intestinal tract. While, virulent NDV (vNDV) have a multibasic amino acid sequence motif at the $\mathrm{C}$-terminus of the F2 protein, and a phenylalanine at the $\mathrm{N}$-terminus of the F1 protein, $112 \mathrm{R} / \mathrm{G} / \mathrm{K}-\mathrm{R}-\mathrm{Q} / \mathrm{K}-\mathrm{K} / \mathrm{R}-$ RF117 and that are cleaved intracellularly by ubiquitous furin-like proteases found in 
most host tissues. This difference in protease activation is the major determinant of disease severity of NDV [5].. Based on sequence analysis of the fusion (F) gene, NDV strains are divided into two distinct classes (I and II). Class I viruses are mostly loNDV found in wild birds, and contain nine genotypes (1-9), but class II viruses were divided into multiple genotypes representing loNDV and vNDV. Almost all virulent NDV strains isolated from wild and domestic birds belong to class II, which can be further subdivided into at least 18 genotypes from I to XVIII [6].

Genotype VII (class II genotype VII) was firstly classified into two subgenotypes: VIIa, which represents viruses that emerged in the Far East and spread to Europe and Asia; and VIIb, which represents viruses that emerged in the Far East and spread to South Africa. Later, genotype VII are classified into other subgenotypes VIIc, $\mathrm{d}$, and e, which represents isolates from China, Kazakhstan and South Africa; and VIIf, g, h, and i, which represent African isolates [5].

In Egypt, the disease had been firstly identified in 1948 [7]. Now days, NDV outbreaks are still frequently occurring in vaccinated poultry flocks, despite the intensive vaccination programs [8-14].

From the above mentioned data our trail is designated in order to characterize the NDV strains responsible for multiple outbreaks in vaccinated Egyptian farms and study its genetic relation with available vaccine and wild viruses in GenBank.

\section{Material and Methods}

Virus Isolates

Four vNDV selected isolates (NDV / Chicken/ EG-MN / NRC / 2015, NDV / Chicken / EG-QU/ NRC/2015, NDV/Chicken / EG-SH / NRC / 2015 and NDV/Chicken/EG-SH2/NRC/2015) isolated and identified by [15] was further passed into allantoic sac at 9-11 days old specific pathogen free (SPF) embryonated chicken eggs (ECE) then incubated at $37 \mathrm{C}$ for 4 days [1].

\section{Haemagglutination (HA) and Hemagglutination Inhibition (HI) test [1].}

Allantoic fluids were tested for haemagglutination (HA) using $1 \%$ chicken RBCs. the HA titer of the allantoic fluid was determined. Field immune sera was used as control positive in $\mathrm{HI}$ test.
Reverse Transcription-Polymerase Chain Reaction (RT-PCR) Extraction of viral RNA

The genomic viral RNA was extracted from harvested HA positive allantoic fluid by using QIAamp viral RNA extraction Kits according to the manufacture's protocol .

Conventional RT-PCR for detection of NDV F-Protein gene [14]

One step RT- PCR was carried using QIAGEN ${ }^{\circledR}$ OneStep RT-PCR kit (QIAGEN, Valencia, CA) according to the manufacturer's instructions. RT-PCR was used for the detection of partial F-gene of vNDV using the following primers: forward 5 -ATGGGCYCCAGACYCTTCTAC-3 and Reverse 5/ -CTGCCA CTGCT AGTT GT G ATAATC-3 that flanks a $535 \mathrm{bp}$ of the F gene of NDV as previously described [16]. Thermal cycling RTPCR conditions included a reverse transcription $50^{\circ} \mathrm{C}$ for $30 \mathrm{~min}$. then an Initial PCR activation step $95^{\circ} \mathrm{C}$ for $15 \mathrm{~min}$. followed by 39 cycles at $94^{\circ} \mathrm{C}$ for $30 \mathrm{sec}$., $55^{\circ} \mathrm{C}$ for $30 \mathrm{sec}$. and $72^{\circ} \mathrm{C}$ for $45 \mathrm{sec}$. then the final extension was performed at $72^{\circ} \mathrm{C}$ for $10 \mathrm{~min}$.

\section{Agarose gel electrophoresis}

The PCR products were separated in $1.5 \%$ agarose gel in TAE buffer stained with ethidium bromide and compared with molecular mass marker (100 bp DNA markers) and visualized by ultraviolet (UV) trans illumination.

\section{Purification of PCR Products from the gel and} gene sequencing:

For gene sequencing, the target bands of specific size were excised from the gel and purified with the QIAquick gel extraction kit (Qiagen, Valencia, CA) according to the manufacturer instructions and The purified DNA was sequenced in an automated ABI 3730 DNA sequence (Applied Biosystems, USA).

\section{Phylogenecity and phylogenetic tree}

The obtained sequences were aligned by the Clustal W method using MEGA V5.05 software. The nucleotide sequences were compared with NDV sequences available in GenBank (Table 1). A phylogenetic tree of aligned sequences was constructed by Boost-trap method. The deduced amino acid sequences were determined to detect the pathotype of isolated NDV. 
TABLE 1. Newcastle disease viruses used in the phylogenetic tree construction.

\begin{tabular}{|c|c|c|}
\hline Reference strain & Accession number & Genotype \\
\hline APMV1/Chicken/US(TX)GB/1948 & GU978777 & Virulent \\
\hline NDV/Chicken/N.Ireland/Ulster/67 & AY562991 & I \\
\hline NDV/Mukteswar & EF201805 & III \\
\hline NDV/US/Largo/71 & AY562990 & $\mathrm{V}$ \\
\hline Pigeon paramyxovirus-1/IT-227/82 & AJ880277 & VI \\
\hline NDV/Chicken/Giza/Egypt/MR0/2012 & JX173098 & VIId \\
\hline NDV/Ostrich/Ismailia/2010 & JN193503 & II \\
\hline NDV/chicken/Egypt/4/2006 & FJ969395 & II \\
\hline NDV/chicken/Egypt/2/2006 & FJ969393 & II \\
\hline Hitchner B1/47 & M24695 & II \\
\hline BeaudetteC/45 & M24697 & II \\
\hline Mexico 468/01 & EU518685 & $\mathrm{V}$ \\
\hline BITPI87079 (Italy) & AY135747 & V \\
\hline Chicken/China/SDYT03/2011 & JQ015297 & VIId \\
\hline Turkey/Israel/111/2011 & JN979564 & VIId \\
\hline Chicken/Israel/174/2011 & JN849578 & VIId \\
\hline Apmv1/Chicken/Jordan/Jo11/2011 & JQ176687 & VIId \\
\hline Chicken/Sudan/03/2003 & GQ258670 & VIId \\
\hline Chicken-2601-Ivory Coast-2008 & FJ772466 & VIIb \\
\hline Avian-1532-14-Mauritania-2006 & FJ772455 & VIIb \\
\hline Chicken-3490-149-Cameroon-2008 & FJ772478 & VIIb \\
\hline NDV/Chicken/Egypt/1/2005 & FJ939313 & Virulent \\
\hline NDV/chicken/Egypt/MR2-1998 & JX193769 & II \\
\hline NDV/Chicken-Behira/Egypt/MR6-2012 & JX193771 & II \\
\hline NDV/VRCLU/Giza/2009 & HQ455810 & II \\
\hline NDV/99/0655/1999/Australia & AY935494 & Virulent \\
\hline NDV/99/PR32/1997/Australia & AY935497 & Virulent \\
\hline NDV/strain NA-1 & DQ659677 & VII \\
\hline NDV/chicken/IS/27/2017 & KY510688 & VII \\
\hline NDV/chicken/IS/929/2016 & KY510684 & VII \\
\hline NDV/chicken/Egypt/Dakahlia28/2016 & KY075891 & VII \\
\hline NDV/Chicken/Egypt/3/2006 & FJ969394 & Virulent \\
\hline Clone_30 & Y18898 & II \\
\hline La_sota & AJ629062 & II \\
\hline VGGA-87 & AF419411 & II \\
\hline Komarov_45-LK & AY170137 & II \\
\hline NDV-Chicken-Egypt-MR3-2003 & JX193770 & II \\
\hline NDV/chicken/Egypt/Damietta9/2016 & KY075882 & VIId \\
\hline NDV/chicken/Egypt/Qualyobia11/2016 & KY075884 & VIId \\
\hline APMV1/chicken/EG-KB2/POD.CU/2015 & KX353698 & VIId \\
\hline APMV1/chicken/EG-SHR/POD.CU/2015 & KX353695 & VIId \\
\hline NDV/chicken/Egypt/Ismailia8/2016 & KY075881 & VIId \\
\hline NDV/chicken/Egypt/El-Arish15/2016 & KY075887 & VIId \\
\hline NDV/Duck/China/Guizhou/ZY/2014 & KU933948 & VIId \\
\hline NDV/Pheasant/H-Israel/2013/746_828 & KF792022 & VIIb \\
\hline
\end{tabular}




\section{$\underline{\text { Results }}$}

Identification of $N D$ virus

The passaged isolates were positive for HA and HI tests. This result of the 4 isolates were confirmed by RT-PCR that indicated the detection F-gene of vNDV result in amplicon 535 bp.

RT-PCR NDV isolates were detected by RTPCR using degenerate primers for the fusion protein gene result in amplicon 535 bp as shown in Fig. 1.

Phylogeny and genetic analysis of the NDV isolates strains

The phylogenetic analysis of partial sequences of the selected four NDV named (NDV/Chicken/EG-MN/NRC/2015,NDV/ Chicken/EG-QU/NRC/2015,NDV/Chicken/ EG-SH/NRC/2015 and NDV/Chicken/EG -SH2/NRC/2015) strains for $\mathrm{F}$ gene showed that the isolated viruses belong to genotype VII (Fig. 2).
The obtained sequences alignment with a Chinese genotype VII (Chicken-ChinaSDYT03-2011) reference strain (retrieved from the Genbank) revealed that the obtained 4 isolates A.A sequence are showing (98-99) \% identity with the genotype VII. As shown in Fig. 3.

The amino acid sequence of fusion protein cleavage site of the isolates carries motif ${ }^{112} \mathrm{RRQKRF}^{117}$ that is consistent with viruses of velogenic strains. Fig.4.

\section{Sequence Submission to Gene Bank}

Sequence of the isolated NDV strains in this study (NDV/Chicken/EG-MN/NRC/2015, NDV/ Chicken/EG-QU/NRC/2015, NDV/Chicken/ EG-SH/NRC/2015 and NDV/Chicken/EG -SH2/ $\mathrm{NRC/2015)}$ was submitted to the Gene Bank by following instructions of the BankIt tool of the Gene Bank http://www.ncbi.nlm.nih.gov/ WebSub/tool=genbank. The submitted sequences to the gene bank were published as verified NDV isolates with accession numbers (MF418017), (MF418018), (MF418019) and (MF418020); respectively.

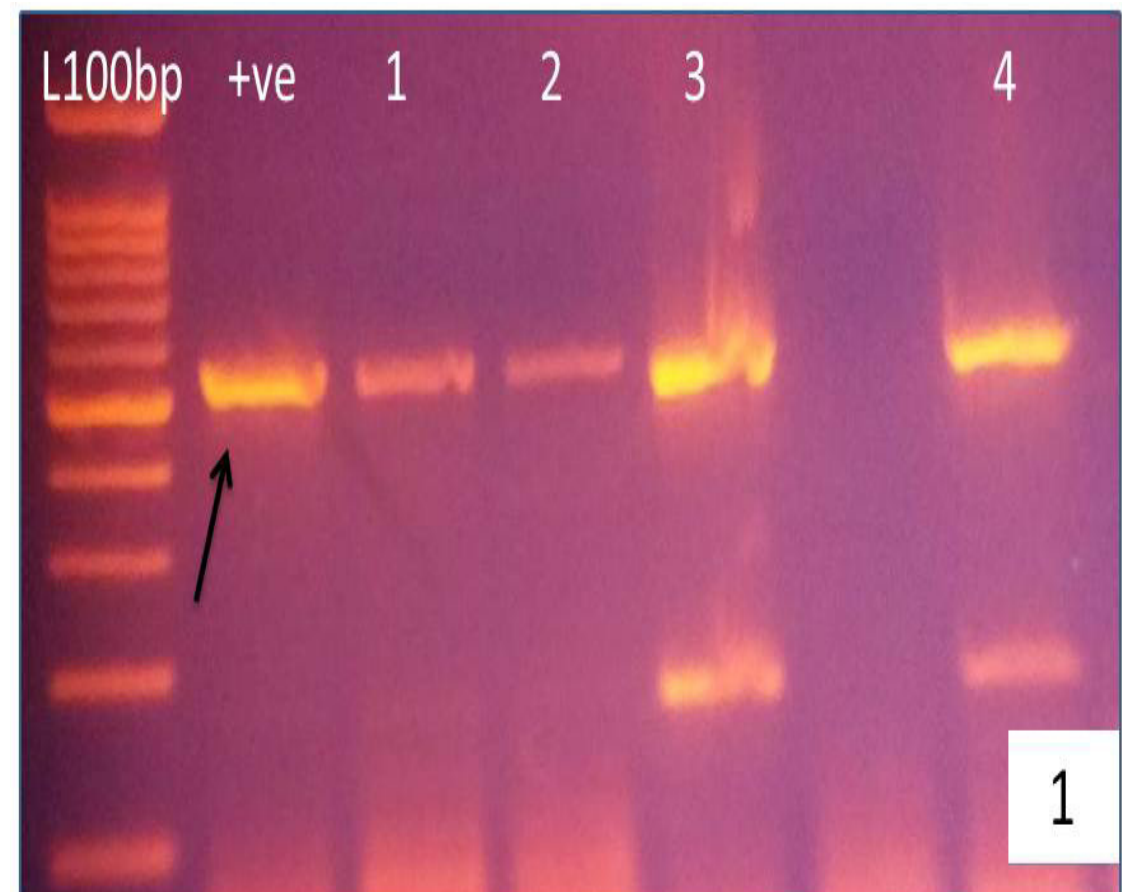

Fig. 1. Agarose Gel Electrophoresis (AGE) pictures showing 535 bp RT-PCR Products of NDV Fusion protein gene. 


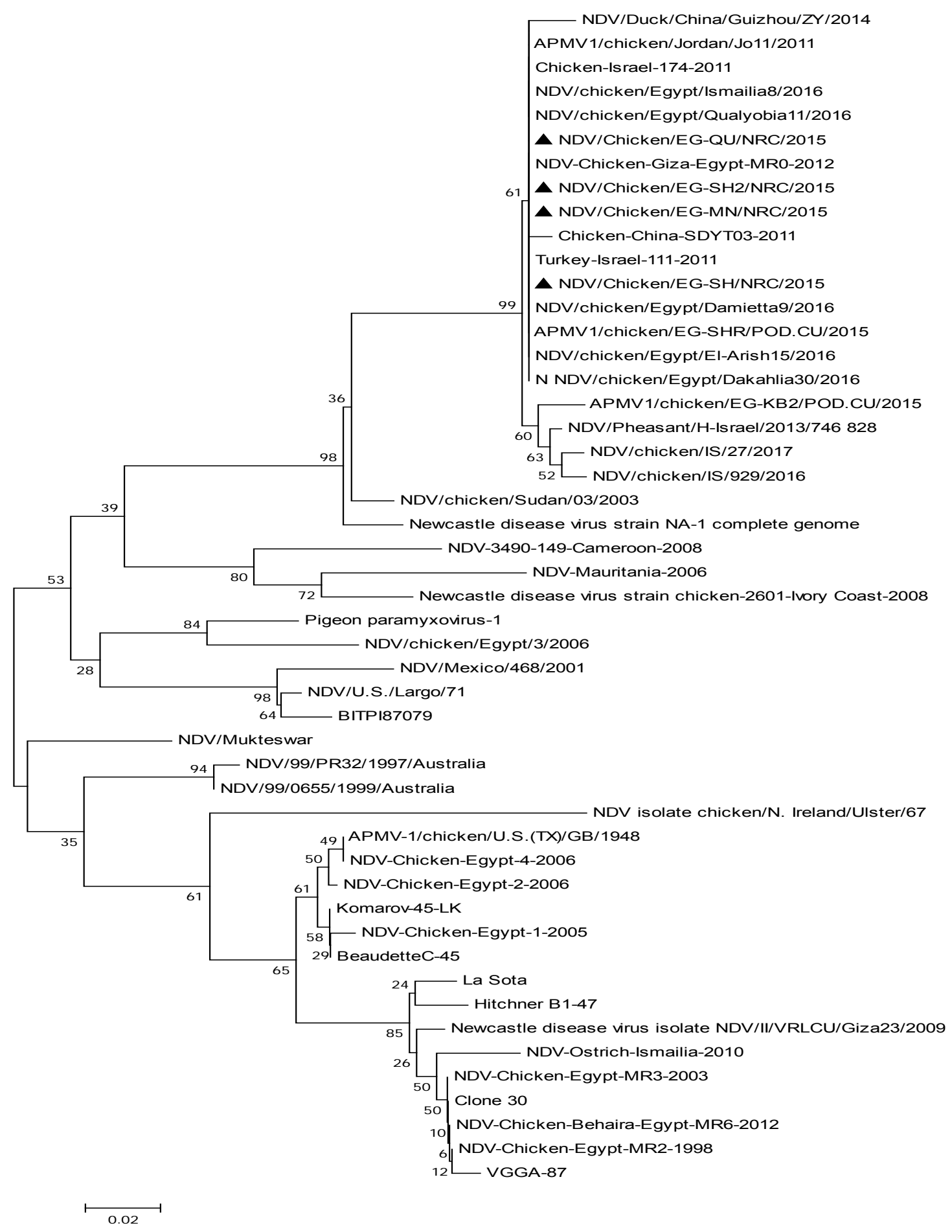

Fig. 2. Phylogenetic tree based on a partial sequence of NDV F gene. showing the relationship between the selected Egyptian NDV isolates in the present study with vaccinal strain and reference NDV strains from gene bank. Black dots refer to viruses isolated in current study. 
NDV/ Chicken/China/SDYT03/2011 NDV/chicken/EG-MN/NRC/2015 NDV/chicken/EG-QU/NRC/2015 NDV/chicken/EG-SH2/NRC/2015

NDV/Chicken/China/SDYT03/2011 NDV/chicken/EG-MN/NRC/2015 NDV/chicken/EG-QU/NRC/2015 NDV/chicken/EG-SH/NRC/2015 NDV/chicken/EG-SH2/NRC/2015

NDV/ Chicken/China/SDYT03/2011 NDV/chicken/EG-MN/NRC/2015 NDV/chicken/EG-MN/NRC/2015 NDV/chicken/EG-SH/NRC/2015 NDV/chicken/EG-SH2/NRC/2015

NDV/Chicken/China/SDYT03/2011 NDV/chicken/EG-MN/NRC/2015 NDV/chicken/EG-QU/NRC/2015 NDV/chicken/EG-SH/NRC/2015 NDV/chicken/EG-SH2/NRC/2015

NDV/Chicken/China/SDYT03/2011 NDV/chicken/EG-MN/NRC/2015 NDV/chicken/EG-OU/NRC/2015 NDV/chicken/EG-SH/NRC/2015. NDV/chicken/EG-SH2/NRC/2015

NDV/ Chicken/China/SDYT03/2011 NDV/chicken/EG-MN/NRC/2015 NDV/chicken/EG-QU/NRC/2015 NDV/chicken/EG-SH/NRC/2015 NDV/chicken/EG-SH2/NRC/2015

NDV/ Chicken/China/SDYTo3/2011 NDV/chicken/EG-MN/NRC/2015 NDV/chicken/EG-QU/NRC/2015
NDV/chicken/EG-SH/NRC/2015 NDV/chicken/EG-SH/NRC/2015
NDV/chicken/EG-SH2/NRC/2015

NDV/Chicken/China/SDYT03/2011 NDV/chicken/EG-MN/NRC/2015 NDV/chicken/EG-QU/NRC/2015 NDV/chicken/EG-SH/NRC/2015 NDV/chicken/EG-SH2/NRC/2015

NDV/Chicken/China/SDYTo3/2011 NDV/chicken/EG-MN/NRC/2015 NDV/chicken/EG-QU/NRC/2015 NDV/chicken/EG-SH/NRC/2015 NDV/chicken/EG-SH2/NRC/2015

NDV/Chicken/China/SDYT03/2011 NDV/chicken/EG-MN/NRC/2015 NDV/chicken/EG-QU/NRC/2015 NDV/chicken/EG-SH/NRC/2015 NDV/chicken/EG-SH2/NRC/2015

NDV/Chicken/China/SDYT03/2011 NDV/chicken/EG-MN/NRC/2015 NDV/chicken/EG-QU/NRC/2015 NDV/chicken/EG-SH/NRC/2015 NDV/chicken/EG-SH2/NRC/2015
ATGGGCTCCAAACСTTCTACCAGGATCCCAGCACСTCTAАTGCTAАTCAC .... . . . . . . . . . . . . . . . . . . . .

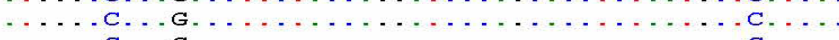
... $\begin{array}{rrrrr}60 & 70 & 80 & 90 & 100\end{array}$ TCGGATTATGCTGACÄTGAGCTGCATCCGTCTGACÄGCTCTCTTGÄCG

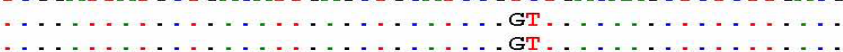
......................... GT

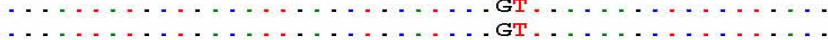

$110 \quad 120 \quad 130 \quad 140 \quad 150$

GCAGGCCCCTTGCAGCTGCAGGAATTGTAGTAACGGGAGATAAGGCAGTC . Т. . . . . . . . . . . . . . . . . . . . . . . . т т $\ldots \ldots \ldots \ldots$ т . . . . . . . . . . . . .
160
170
180
190
200

ÄTGTATÄCACCTCGTCTCAGACAGGGTCAATCATAGTCAÄTTGCTCCC . .
210
220
230
240
250

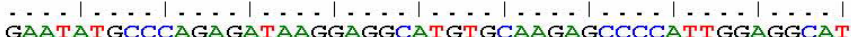
$\ldots \ldots \ldots \ldots \ldots$

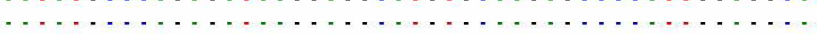

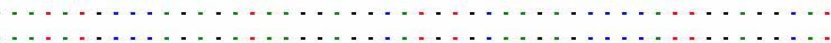

260 270 280 290

ATAACAGgACACTGACTACTCTGCTCACTCCTCTTGGTGACTCCATCCGC ... . . . . . . . . . . . . . . . . . . . . .

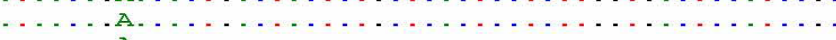

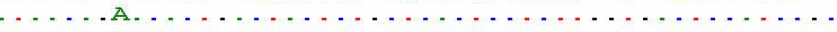

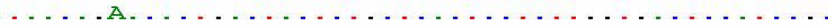
310
320
330
340
350

AAGATCCAAGGGTCI $\ldots$ - .

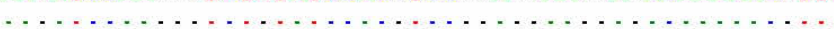
$\ldots \ldots$

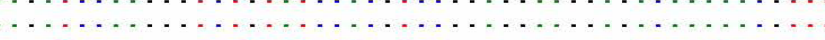

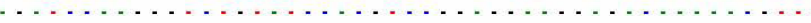
360
370
380
390
400

TATAGGTGCTGTTATTGGCAGTGTAGCTCTTGGGGTTGCAACÄCGGCAC . . . . . . . . . . . . . . . . . . . . . . . . $\ldots \ldots$. . . . . . . . . . . . . . . . . . . .
410
420
430
440
450

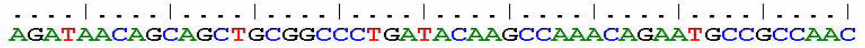

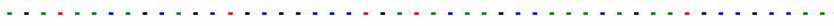

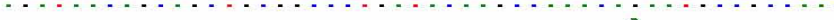

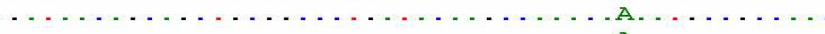

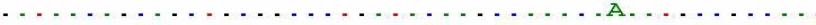

$460 \quad 470 \quad 480 \quad 490 \quad 500$

АТССtCCGGCTTAAGgÄGAGCATTGCTGCAACCAATGÄGCTGTGCATGA

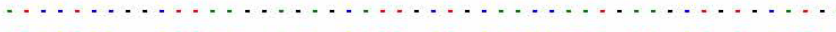

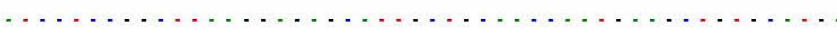
$\ldots \ldots \ldots \ldots$

$$
510 \quad 520 \quad 530
$$

AGTCACCGACGGATTATCACÄACTATCÄGTGCAGT $\ldots \ldots$ T . . . . . . . . . . . . . . . . . .

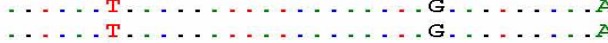

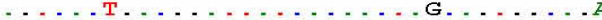

Fig.3. Alignment of the obtained isolates nucleotides sequences with reference isolate

Egypt. J. Vet. Sci. Vol. 48, No.1 (2017) 


\begin{tabular}{|c|c|c|c|c|c|c|c|c|c|c|c|c|c|c|c|c|c|c|c|c|c|c|}
\hline$\vec{\nabla}$ & $\stackrel{\Xi}{8}$ & 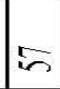 & $\stackrel{\nabla}{\circ}$ & $\stackrel{\overrightarrow{0}}{\circ}$ & $\stackrel{\nabla}{\circ}$ & $\vec{\circ}$ & 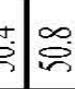 & $\begin{array}{l}8 \\
:\end{array}$ & $\tilde{E}$ & $\frac{7}{5}$ & 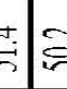 & 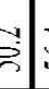 & $\vec{\circ}$ & $\approx$ & $\lesssim$ & $\mathcal{Z}$ & 워 & $\begin{array}{l}0 \\
\infty \\
\infty\end{array}$ & 㝘 & $\vec{\sigma}$ & 过 & \\
\hline$\nexists$ & 迹 & $\sqrt{\infty}$ & $\stackrel{\vec{s}}{*}$ & $\stackrel{\vec{\infty}}{\Delta}$ & $\vec{\infty}$ & $\ddot{\infty}$ & $\vec{\Omega}$ & i & 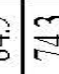 & $\frac{0}{\circ}$ & 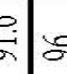 & 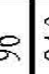 & के & $\vec{\infty}$ & $\frac{7}{\infty}$ & $\approx$ & $\infty$ & इ & $\ddot{g}$ & 2 & & 衣 \\
\hline 으 & $\frac{3}{\infty}$ & $\vec{\infty}$ & 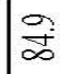 & $\frac{9}{\infty}$ & $\frac{a}{\infty}$ & $\frac{a}{\infty}$ & : & $\frac{3}{5}$ & $\tilde{F}$ & $\sum \lesssim$ & i. & 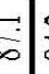 & $\vec{\Xi}$ & 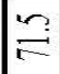 & $\sqrt{\infty}$ & $\stackrel{\infty}{\infty}$ & જี & $\infty$ & $\infty$ & & $\stackrel{2}{\infty}$ & $\vec{g}$ \\
\hline$\infty$ & $\vec{\infty}$ & $\vec{\delta}$ & 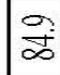 & $\frac{9}{\infty}$ & $\frac{9}{\infty}$ & $\frac{2}{\infty}$ & : & \begin{tabular}{l|l}
$\sim$ \\
\end{tabular} & $E$ & $\frac{0}{\sigma}$ & 5 & $\frac{3}{3}$ & ळై & $\overrightarrow{\dot{\infty}}$ & i̊ & 5 & $\infty$ & $\infty$ & & $\infty$ & $\overrightarrow{8}$ & 兼 \\
\hline$\Xi$ & 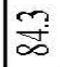 & $\tilde{\infty}$ & 急 & 令 & $\frac{9}{\infty}$ & ; & : & ज怘 & $\tilde{F}$ & $\frac{0}{\sigma}$ & 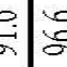 & : & $\stackrel{3}{\$}$ & $\stackrel{3}{\infty}$ & $\sqrt{\infty}$ & 莆 & के & & 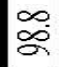 & $\infty$ & 麦 & 过 \\
\hline 0 & $\frac{3}{\infty}$ & $\vec{\infty}$ & సं & $\infty$ & - & $\vec{\infty}$ & 寉 & b) & 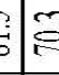 & $\bar{\infty}$ & 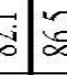 & 8 & $\frac{2}{\infty}$ & $\vec{\varepsilon}$ & $\vec{\infty}$ & $\vec{\infty}$ & & $\infty$ & 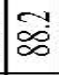 & శี่ & $\infty$ & \%े \\
\hline$\simeq$ & $\approx$ & 鬲 & $\stackrel{\Xi}{\circ}$ & $\stackrel{\Xi}{\circ}$ & $\stackrel{\bullet}{\circ}$ & $\stackrel{i}{\circ}$ & $\int_{0}^{\infty}$ & $\dot{3} \overline{3}$ & s: & $\infty_{0}^{\infty}$ & $\stackrel{\infty}{0} \mid \infty$ & $\begin{array}{l}\infty \\
0 \\
0\end{array}$ & $\approx$ & 2 & 突 & & $\stackrel{\infty}{\infty}$ & 8 & 5 & $\stackrel{\circ}{\circ}$ & 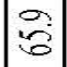 & 그 \\
\hline 士 & ঠे & $\ddot{2}$ & E. & 동 & 5 & 5 & $\stackrel{\circ}{\circ}$ & I & $\approx$ & $\dot{s}: \stackrel{0}{\sharp}$ & $\stackrel{\Omega}{2}$ & & ㅎ. & 5 & & 突 & $\frac{0}{\infty}$ & $\approx \tilde{s}^{\circ}$ & 兽 & $\approx$ & $\frac{7}{\infty}$ & $\stackrel{\infty}{\circledR}$ \\
\hline 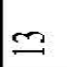 & $\frac{2}{6}$ & $\frac{7}{8}$ & $\frac{1}{6}$ & 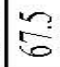 & $\approx$ & $\approx$ & $\frac{1}{0} \stackrel{\infty}{\stackrel{0}{ \pm}}$ & if & $\tilde{E}$ & $\stackrel{\infty}{d}$ & $\stackrel{ \pm}{0}$ & & 5 & & 5 & 2 & $\stackrel{\beta}{\beta}$ & $\frac{3}{\infty}$ & $\overrightarrow{\tilde{S}}$ & $\cong$ & $\infty$ & $\stackrel{\infty}{\infty}$ \\
\hline$\simeq$ & 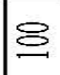 & $\mid \infty$ & 2 & $\mathscr{8}$ & $\mathscr{B}$ & 2 & $\infty$ & 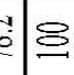 & $\bar{C}$ & $\infty$ & $\vec{e} \mid \frac{0}{2}$ & & & 5 & ন্ & $\approx$ & $\stackrel{2}{\infty}$ & $\vec{F}$ & ळె' & $\frac{m}{\infty}$ & 产 & $\stackrel{\overrightarrow{0}}{\circ}$ \\
\hline$\equiv$ & $\frac{a}{\infty}$ & $\bar{\infty}$ & $\approx$ & $\approx$ & 3 & 3 & $\frac{1}{8}$ & $\frac{a}{\infty}$ & $\stackrel{\infty}{\circ}$ & $\ddot{\infty}$ & 8 & & 胥 & 2 & ) & $\mid \begin{array}{l}\infty \\
8 \\
8\end{array}$ & 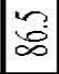 & $\ddot{2}$ & בุ & $\vec{\infty}$ & 2 & $\widetilde{\ominus}$ \\
\hline$\varrho$ & $\stackrel{\infty}{\infty}$ & 2 & $\tilde{\infty}$ & $\infty$ & $\infty$ & $\infty$ & $\begin{array}{lll} \\
0\end{array}$ & $\infty$ & $\bar{F}$ & & $\tilde{a}$ & & $\infty$ & $\stackrel{\infty}{+}$ & $\because$ & $\infty$ & $\overrightarrow{\text { si }}$ & $\frac{0}{-1}$ & $\frac{0}{2}$ & $\mid \begin{array}{c}0 \\
\infty \\
\infty\end{array}$ & 이 & $\vec{\nabla}$ \\
\hline a & $\vec{\infty}$ & $\vec{\infty}$ & Е & 定 & $\vec{\infty}$ & क & $\bar{c}$ & $\vec{\infty}$ & & $\bar{E}$ & $\vec{\Sigma} \mid \stackrel{\curvearrowright}{F}$ & & $\vec{\infty}$ & $\ddot{\Omega}$ & $\approx$ & $\cong$ & $\hat{e}$ & $\widetilde{\mathrm{s}}$ & $\vec{\sigma}$ & $\overrightarrow{\mathrm{e}}$ & $\underset{2}{2}$ & 3 \\
\hline$\infty$ & $\Xi$ & $\infty$ & $\ddot{\mathscr{O}}$ & $\ddot{2}$ & $\ddot{2}$ & $\mathscr{B}$ & 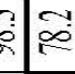 & & $\infty$ & 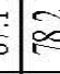 & $\ddot{e})$ & & $\Xi$ & $\approx$ & ১: & $\approx$ & $\stackrel{3}{\infty}$ & $\frac{3}{\infty}$ & $\tilde{\infty}$ & $\frac{3}{\infty}$ & 商 & $\stackrel{+}{\circ}$ \\
\hline$m$ & $\infty$ & 2 & $\infty$ & $\infty$ & $\infty$ & $\infty$ & & ळ & $\bar{F}$ & 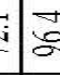 & $\stackrel{8}{8}$ & & $\infty$ & $\stackrel{\infty}{ \pm}$ & $\stackrel{0}{2}$ & $\bigotimes_{0}^{\infty}$ & 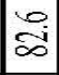 & $\vec{\Omega}$ & $\bar{\Omega}$ & ळే & $\vec{\Omega}$ & $\stackrel{\infty}{\stackrel{8}{\circ}}$ \\
\hline 0 & $\ddot{g}$ & $\stackrel{\vec{S}}{5}$ & $\S$ & $\S$ & 8 & & $\widetilde{\infty}$ & 0 & $\bar{\infty}$ & $\infty$ & 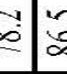 & & $\ddot{2}$ & $\approx$ & S: & $\stackrel{\Xi}{\circ}$ & $\vec{\infty}$ & 过 & 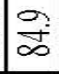 & 高 & $\ddot{\infty}$ & $\stackrel{\overrightarrow{0}}{\circ}$ \\
\hline in & $\ddot{g}$ & కñ & 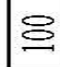 & 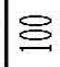 & & $\S$ & $\vec{\rho}$ & $\dot{2}$ & $\bar{\infty}$ & 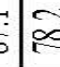 & $\ddot{e}$ & & $\ddot{2}$ & $\approx$ & Е & $\stackrel{+}{\circ}$ & $\overrightarrow{\text { s }}$ & $\frac{a}{\infty}$ & $\frac{9}{\infty}$ & $\frac{a}{\infty}$ & $\stackrel{+}{\infty}$ & $\stackrel{7}{\circ}$ \\
\hline$\rightarrow$ & $\ddot{2}$ & בֶ: & $\Xi$ & & $\S$ & 8 & 3 & $\dot{2}$ & $\bar{\infty}$ & $\infty$ & $e$ & & $\ddot{8}$ & $\approx$ & E. & $\stackrel{t}{\circ}$ & $\overrightarrow{\text { s }}$ & $\frac{j}{\infty}$ & $\frac{9}{\infty}$ & के & $\underset{\infty}{\infty}$ & $\stackrel{\nabla}{\circ}$ \\
\hline$m$ & $\ddot{2}$ & $\frac{1}{2}$ & & 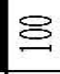 & 8 & 8 & $2 \infty$ & 02 & $\vec{\infty}$ & $\infty$ & $\ddot{e} \mid \ddot{2}$ & & $\ddot{2}$ & $\approx$ & E. & $\stackrel{\nabla}{\circ}$ & $\overrightarrow{\text { si }}$ & 迹 & 产 & 感 & 灾 & $\stackrel{\overrightarrow{0}}{\circ}$ \\
\hline$\sim$ & $\infty$ & & בี & בั & 点 & 䓅 & 12 & $1 \%$ & $\dot{\alpha}$ & 5 & $\Rightarrow 2$ & & $\stackrel{\infty}{\infty}$ & $\stackrel{7}{8}$ & 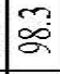 & 豈 & $\overrightarrow{\dot{\infty}}$ & $\stackrel{\sim}{\infty}$ & $\overrightarrow{\text { ¿े }}$ & $\vec{\infty}$ & $\vec{\infty}$ & in \\
\hline 一 & & 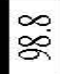 & $\ddot{z}$ & $\ddot{g}$ & 3 & 8 & $8 \infty$ & 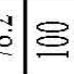 & $\vec{\alpha}$ & $\infty$ & $\infty$ & & $\Theta$ & 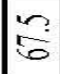 & ন্: & $\ddot{3}$ & $\frac{2}{\infty}$ & $\frac{7}{\infty}$ & ळூ & $\frac{7}{\infty}$ & के & $\vec{\circ}$ \\
\hline & 一 & -1 & $m$ & 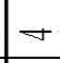 & in & 10 & $01-$ & $-\infty$ & 0 & $\subseteq$ & $2=$ & $=$ & $\simeq$ & 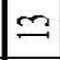 & 三 & $\because$ & 10 & $=$ & $\infty$ & a & 이 & $\approx$ \\
\hline $\begin{array}{l}\text { 总 } \\
\text { 㴔 }\end{array}$ & 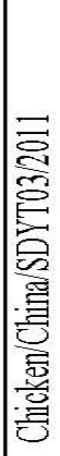 & 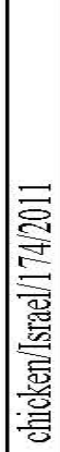 & 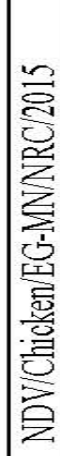 & 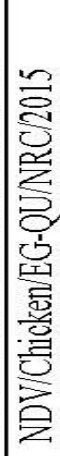 & 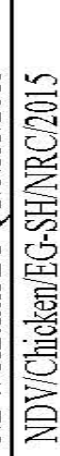 & 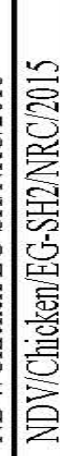 & 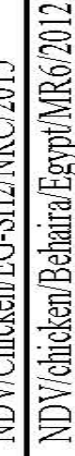 & 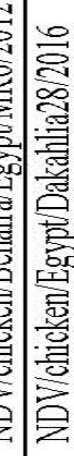 & 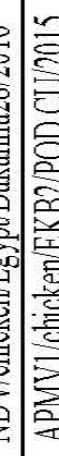 & 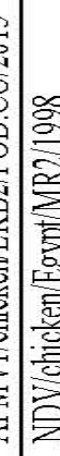 & 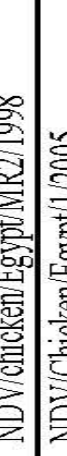 & 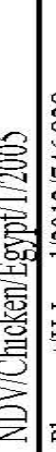 & 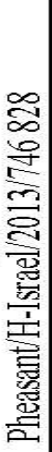 & 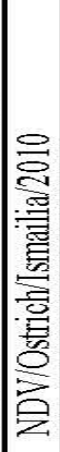 & 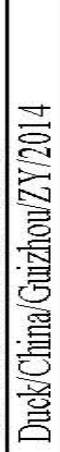 & 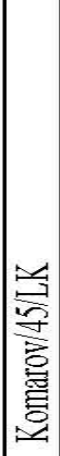 & 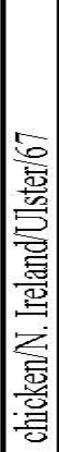 & 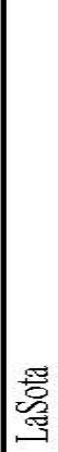 & 兽 & 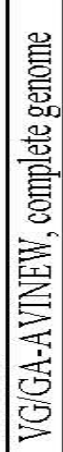 & 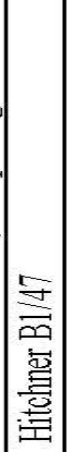 & 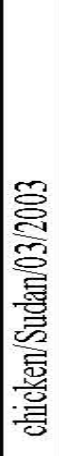 \\
\hline
\end{tabular}




\section{Discussion}

Newcastle disease virus considered the most predominant avian viral diseases affecting poultry industry in Egypt causing high economical losses , although intensive vaccination programmes carried out in field [17]. This may be due to genetic diversity among NDV strains in last years [5] resulting in field outbreaks or emerging of new pathotype resulting in severs infection [10]. So, the isolation and pathotyping of NDV from outbreaks among chickens is a critical for the control of NDV and vaccination evaluation [13].

Molecular identification by RT-PCR revealed that; our isolate is Newcastle field virus this result obtained from positive sample from clinical cases after primary isolation in specific pathogen free embryonated chicken egg (SPF-ECE). Many researcher used RT-PCR for isolation of Newcastle disease as reliable method for detection of positive cases $[18,19]$ which considered accurate method for detection of NDV.

For relatedness detection of isolated NDV field isolates sequencing is carried out,This method is a rapid and reliable method for NDV pathotyping as compared to the mean death time, the intravenous pathogenicity index or intracerebra pathogenicity index tests [20,21]. Results confirmed that all strains of this study shared the cleavage site motif112 RRQKRF117 which is characteristic for vNDV strains and these results also revealed that our isolated strain belonged to class II genotype VIId which considered velogenic strain causing severe outbreaks in china [22] and middle east [23].

The recent vNDV isolates in this study (genebank acc. no.MF418017, MF418018, MF418019 and MF418020) showed complete identity to each other with $100 \%$ identity and 96\%-99\% identity with other vNDV strains present on genbank.

It is concluded that, NDV isolates circulating among chickens are virulent ( Genotype VIId) and associated with outbreaks in poultry farms and is responsible for severe economic losses.

For prevention control of such virus it is recommended for further protective trial using different vaccination regimes for choosing the best protocol in order to control such pathotype under our Egyptian field conditions which needs further investigations.

\section{$\underline{\text { References }}$}

1. OIE (2012) Newcastle disease. Manual of Diagnostic Tests and Vaccines for Terrestrial Animals. Chapter 2.3.14. http://www.oie.int/ international-standardsetting/terrestrial-manual/access-online.

2. ICTV (2012) King, A., Lefkowitz, E., Adams, M.I. and Carstens, E.B. Virus taxonomy: ninth report of the International Committee on Taxonomy of Viruses. Academic Press, London, United Kingdom.

3. de Leeuw, O. and Peeters, B. (1999) Complete nucleotide sequence of Newcastle disease virus: evidence for the existence of a new genus within the subfamily Paramyxovirinae. Journal of General Virology, 80, 131-136.

4. Alexander, D.J. and Senne, D.A. (2008) Newcastle disease and other avian paramyxoviruses. In: Y. M. Saif, A. M. Fadly, J. R. Glisson, L.R. McDougald, L. K. Nolan and D.E. Swayne (Ed.), "Diseases of Poultry", $12^{\text {th }}$ ed., Ames, IA: Iowa State University Press, pp. 75-116.

5. Miller, P.J., Decanini, E.L and Afonso, C.L. (2010) Newcastle disease evolution of genotypes and the related diagnostic challenges. Infect. Genet Evol., 10, 26-35.

6. Snoeck, C.J., Owoade, A.A., Hymann, E.C., Alkali, B.R., Okwen, M.P., Adeyanju, A.T., Komoyo, G.F., Nakoun, E., Le Faou, A. and Muller, C.P. (2013) High genetic diversity of Newcastle disease virus in poultry in West and Central Africa: cocirculation of genotype XIV and newly defined genotypes XVII and XVIII. J Clin Microbiol., 51 (7), 2250-60.

7. Daubney, R. and Mansy, W (1948) The occurrence of Newcastle disease in Egypt. J. Comparat. Pathol. Therap., 58, 189-200.

8. Abdel-Moneim, A.S., El-Sawah, A.A. and Kandil, M.A. (2006) Characterization of variant strain of Newcastle disease virus in Egypt. BS. Vet. Med. J., 16, 12-17.

9. Mohamed, M.H.A., Kumar, S., Paldurai, A., Megahed, M.M., Ghanem, I.A., Lebdah, M.A. and Samal, S.K. (2009) Complete genome sequence of a virulent Newcastle disease virus isolated from an outbreak in chickens in Egypt. Virus Genes, 39, 234-237. DOI 10.1007/s11262-009-0385-7.

10. Radwan, M.M., Darwish, S.F., El-Sabagh, I.M., El-Sanousi, A.A and Shalaby, M.A. (2013) Isolation and molecular characterization of Newcastle disease virus genotypes II and VIId in Egypt between 2011 and 2012. Virus Genes, 47, 311-316. 
11. Hussein, H.A., Emara, M.M. and Rohaim, M.A (2014) Molecular characterization of Newcastle Disease Virus Genotype VIID in Avian influenza H5 N1 Infected Broiler Flock in Egypt. Internat. $J$. of Virol., 10 (1), 46 - 54.

12. Nabila Osman, Sultan, S., Ahmed, I.A., Ibrahim, R.S. and Sabra, M. (2014) Isolation and Pathotyping of Newcastle Disease Viruses from Field Outbreaks among Chickens in the Southern Part of Egypt 2011-2012. Global Veterinaria, 12 (2), 237-243

13. Awad, A.M., Sedeik, M.E. and Abdel kariem, A. A. (2015) Isolation, Molecular Characterization And Pathotyping Of Newcastle Disease Viruses From Field Outbreaks Among Broiler Flocks In Egypt From 2014-2015. Internat. J. of Current Res., 7 (2), 12925-12934. ISSN: 0975-833X.

14. El-Bagoury, G.F., Samar F. El-Adaway, ElHabbaa, A.S. and Suzan S. El-Mahdy (2015) Isolation, identification and pathotyping of Newcastle disease virus from chickens in Egypt. Benha Vet. Med. J., 29 (1), 196-204.

15. Hagar M. Ahmed, Amer, M. M., Elbayoumi, Kh. M., Kutkat, M.A. (2017) Isolation and identification of Genotype VII of Newcastle disease virus from chicken flocks in six Egyptian Governorates. Accepted in Specialty J of Medical Res and Health Sci., Vol 2, Issue1.

16. Seal, B. S. (1995) Analysis of matrix protein gene nucleotide sequence diversity among Newcastle disease virus isolates demonstrates that recent disease outbreaks are caused by viruses of psittacine origin. Virus Genes, 11, 217-224.
17. Sabra, M. H. (2013) molecular characterization of Newcastle disease virus isolated from chickens in south valley provinces. M.Sc. Thesis, South Valley University, Qena, Egypt.

18. Wang, Z., Vreede, F.T., Mitchell, J.O., Viljoen, G.J (2001) Rapid detection and differentiation of Newcastle disease virus isolates by a triple one-step RTPCR. Onderstepoort J. Vet. Res., 68, 131-134.

19. Creelan, L.J., Graham, D.A. and Mc Cullough, Samuel J. (2002) Detection and differentiation of pathogenicity of avian paramyxovirus serotype 1 from field cases using one-step reverse transcriptase polymerase chain reaction. Avian Pathology, 31 (5), 493-499.

20. De Battisti, C., Salomoni, A., Ormelli, S., Monne, I., Capua, I. and Cattoli, G. (2013) Rapid pathotyping of Newcastle Disease Virus by pyrosequencing. J. Virol. Method, 188, 13-20.

21. Ganar, K., Das, M., Sinha, S., Kumar, S.(2014) Newcastle disease virus: current status and our understanding. Virus Res., 184, 71-81.

22. Liu, X.F., Wan, H.Q., Ni, X.X., Wu, Y.T. and Liu, W.B. (2003) Pathotypical and genotypical characterization of strains of Newcastle disease virus isolated from outbreaks in chicken and goose flocks in some regions of China during 1985-2001. Archives of Virol., 148, 1387-1403.

23. Khan, S.T., Rehmani, Rue. C., Miller, P. and Afonso, C.L. (2010) Phylogenetic and pathological characterization of Newcastle disease virus isolates from Pakistan. J. Clin. Microbiol., 48, 1892-1894.

(Received 15/6/2017; accepted 7/ 8/2017) 


\section{ستريد وتبطيلس مصرية الوراثي السابع من فيروس مرض النيوكاسل من قطعان الاجاج في

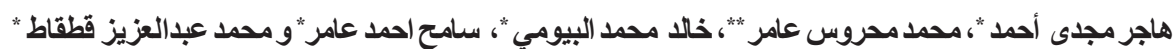

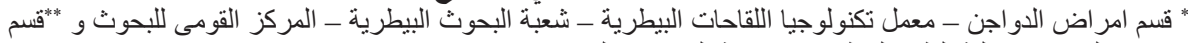 امر اض الدو اجن - كلبة الطب البيطري - جامعة القاهرة - القاهرة - مصر.}

مرض النيو كاسل هو مرض معدى جدا في الدجاج و الديوك الرومي وأحد أهم أمر اض الدو اجن في العالم. تسبب التبات

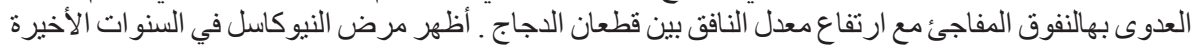

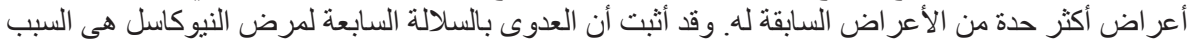

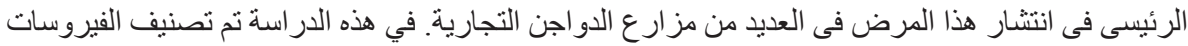

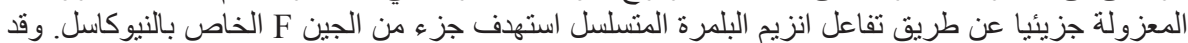

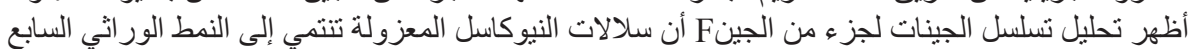

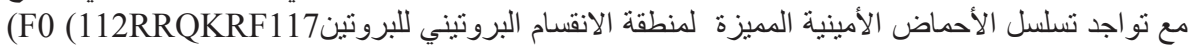

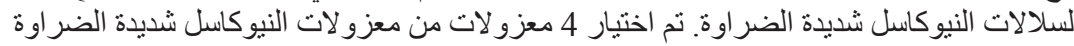
NDV/Chicken/EG-MN/NRC/2015， NDV/Chicken/EG-QU/NRC/2015, NDV/)

(Chicken/EG-SH/NRC/2015 and NDV/Chicken/EG-SH2/NR/2015 MF418017), (MF418018), (MF418019)) وتسجيلها على بنك الجينات تحت الارقام التالية (MF418020 () بالترتبب. (M) (

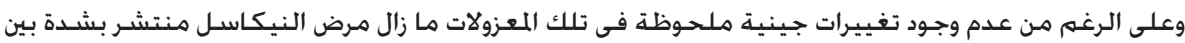

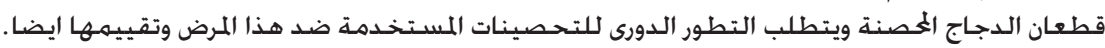

الكلمات الدالة: التعرف على ، التسلسل الجينى ، فيروس مرض النيوكاسل ، الدو اجن. 